\title{
107. Time Lag in the Emission of Spectral Lines Excited in Vacuum.
}

\author{
By Hantaro NAGAOKA and Tetsugoro Futagami.
}

Institute of Physical and Chemical Research, Tokyo.

(Rec. July 11, 1928. Comm. by H. NAGAOKA, M.I.A., July 12, 1928.)

We have already sketched the emission of spectral lines excited by means of disruptive discharge in air of a condenser battery of 1.3 microfarad capacity. The minute retardation in the emission of different lines was apparent in many experiments, and short notice was already given. The photograms taken in vacuum by applying strong magnetic field revealed an easy means of distinguishing arc and spark lines. An analogous result was obtained with moving film.

A discharge vessel was constructed of a brass cylinder with a window covered with quartz plate; the electrodes were led inside along the axes of thick ebonite rods fitting into the cylinder, and the metal to be tested attached at the ends of the electrodes in loose contact. Two large oil pumps connected in series served to evacuate the vessel ; the discharge took place when the vacuum reached a state of producing green fluorescence. The light was received on the slit $(0.02 \times 0.5 \mathrm{~mm}$.) of a quartz spectrograph and the spectrum projected on a film wrapped on an aluminium drum, rotating with peripheral speed of $100 \mathrm{~m}$. per second. The period of a half-oscillation of the discharge was about $10^{-5}$ sec.

During the initial phase of the discharge, the gases remaining in the cylinder give rise to emission of air lines. Many spark lines are emitted, but the arc lines do not appear at the initial moment. It takes a half-oscillation or more before the lines appear ; they go on increasing in intensity, reaches a maximum and gradually fade away. The lag is illustrated in Fig.'s 1 and 4 for $\mathrm{Al}$ and $\mathrm{Cu}$ resp. For comparison, spectra in air (Fig.'s 3 and 6) are also given; they indicate that the lines are stronger and the reversal more prominent in air than in vacuum. Fig.'s 2 and 5 are spectrograms taken on stationary film by discharge in vaccum.

1) Nagaoka, Nukiyama a. Futagami: Proc. Imp. Acad. 3 (1927) 208, 212, 258, $262,319,324,330,392,398,403,409,415,499$.

2) Nagaoka a. Futagami : Proc. Imp. Acad. 3 (1927) $647 ; 4$ (1928) 195. 
As an instance, following lines of $\mathrm{Cu}$ showing the time lag are cited with their series relations.

$\begin{array}{llll}4531 & 2^{2} \mathrm{P}_{2}-3^{2} \mathrm{~S}_{1} & 4023 & 2^{2} \mathrm{P}_{1}-4^{2} \mathrm{D}_{2} \\ 4509 & { }^{4} \mathrm{~F}_{3}-{ }^{4} \mathrm{D}_{1} & 3602 & { }^{4} \mathrm{D}_{4}-{ }^{4} \mathrm{D}^{1}{ }_{4} \\ 4480 & 2^{2} \mathrm{P}_{1}-3^{2} \mathrm{~S}_{1} & 3599 & { }^{2} \mathrm{P}_{2}-5^{2} \mathrm{~S}_{1} \\ 4378 & { }^{4} \mathrm{P}_{2}-{ }^{4} \mathrm{D}_{3} & 3308 & \\ 4275 & { }^{4} \mathrm{P}_{3}-{ }^{4} \mathrm{D}_{4} & 3291 & 4 \overline{\mathrm{D}}_{3}-{ }^{4} \mathrm{G}_{4} \\ 4178 & { }^{4} \mathrm{P}_{3}-{ }^{4} \mathrm{D}_{3} & 3274 & 1^{2} \mathrm{~S}-2^{2} \mathrm{P}_{1} \\ 4063 & 2^{2} \mathrm{P}_{2}-4^{2} \mathrm{D}_{2}, 2^{2} \mathrm{P}_{2}-4^{2} \mathrm{D}_{3} & 3248 & 1^{2} \mathrm{~S}-2^{2} \mathrm{P}_{2}\end{array}$

The last two lines show strong reversal in air, but not in vacuum.

The following observation seems to explain the general feature of the phenomenon. The whole volume of the vessel including the connecting tube brightens up at the discharge, showing that the residual gas is ionised already on the first passage of the current. We have strong evidence of the disintegrated particles being strongly electrified at the initial moment of the discharge, as noticed in our communications on the sputtering and electric explosions ${ }^{1)}$. The emission of spark lines in the initial stage is a natural consequence of the process going on during the discharge. In the evacuated space, the electrons are not sufficiently numerous to excite the arc lines, but gradually increase with successive oscillations. In the mean time, some of the ionized atoms recombine and are freshly excited, as shown by the gradual transition in the intensity of lines. By decreasing the pressure still further, the lag will become longer. From the photograms it is probable that the change in energy levels of electrons in ionized atoms occurs almost simultaneously with the process of ionisation, to which state the liberated atoms mostly enter at the first discharge.

On comparing the spectra obtained by stationary film with those by the moving, we find all the lines which appeared during the discharge on both of them, but in the former we cannot tell which of the lines were excited during the initial passage of the current or after it, being a promiscuous assemblage of all the lines of the spectra without any regard to the order of their appearance. The present experiment shows that the history of the excitation of lines is of importance in studying the mechanism of emission.

1) Nagaoka a. Futagami: Sci. Papers Inst. Phys. Chem. 8 (1928) 269.; Proc. Imp. Acad. 3 (1927) 499, 643 ; 4 (1928) 106, 201. 


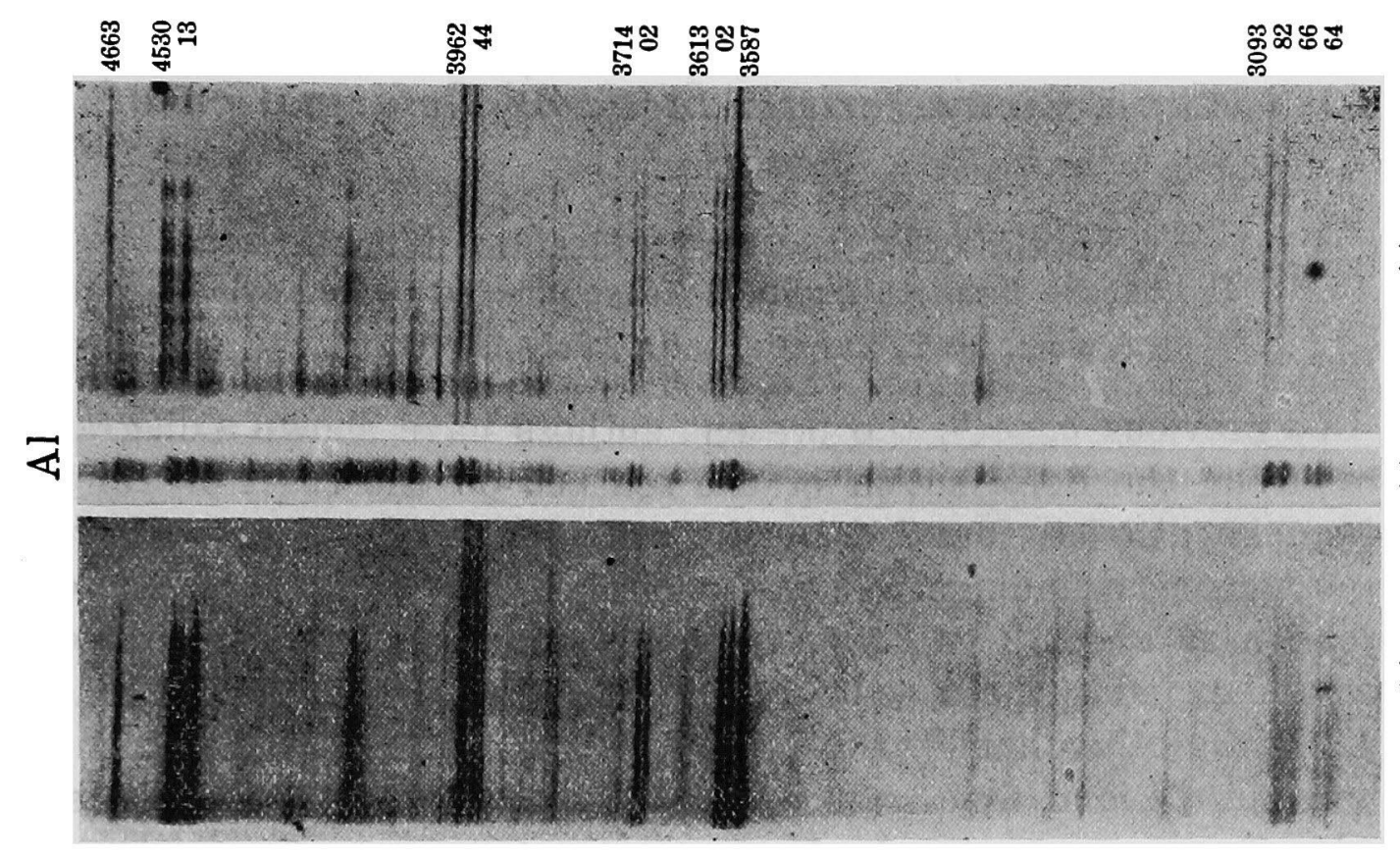

Fig. 1

Fig. 3

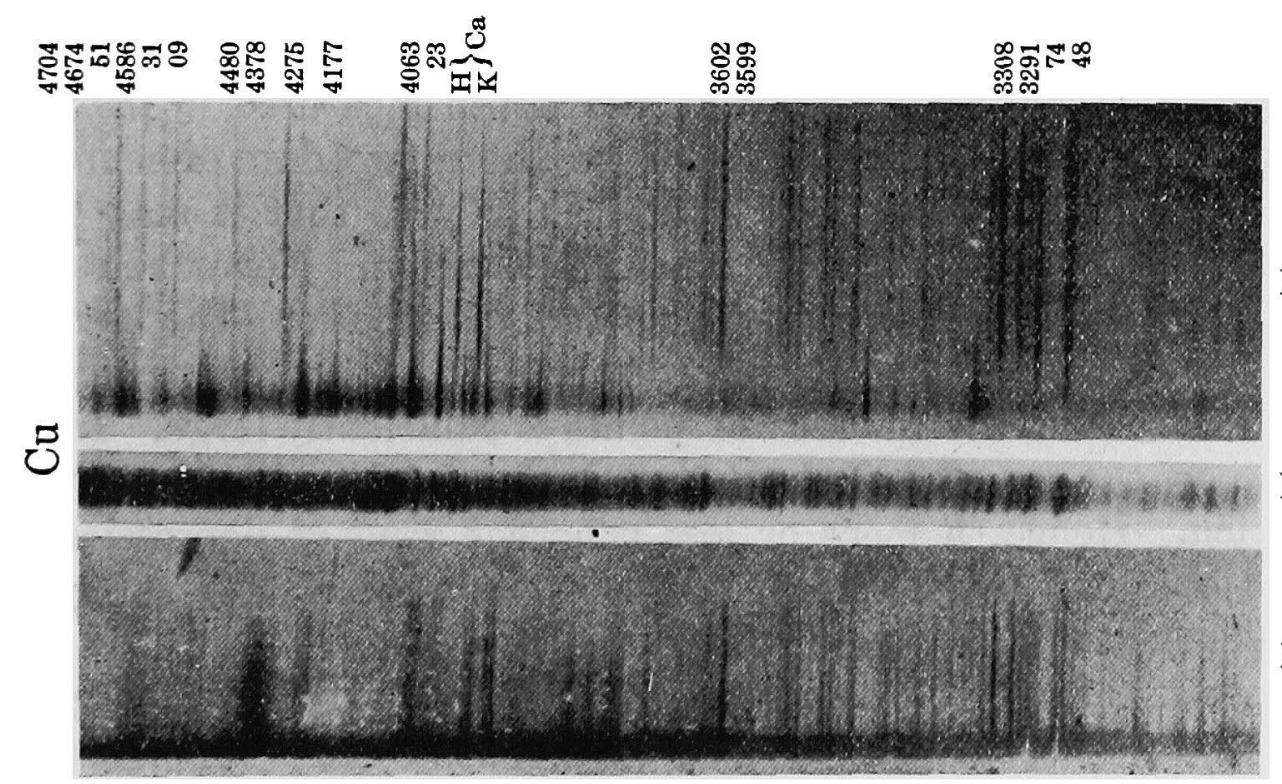

Fig. 4

Fig. 6 